\title{
Quantum Billiards with Surface Scattering: Ballistic Sigma-Model Approach
}

\author{
Ya. M. Blanter ${ }^{a}$, A. D. Mirlin ${ }^{b, *}$, and B. A. Muzykantskii ${ }^{c}$ \\ ${ }^{a}$ Département de Physique Théorique, Université de Genève, CH-1211 Genève 4, Switzerland \\ ${ }^{b}$ Institut für Theorie der Kondensierten Materie, Universität Karlsruhe, 76128 Karlsruhe, Germany \\ ${ }^{c}$ Department of Physics, University of Warwick, CV4 7AL Coventry, UK
}

(December 22, 1997)

\begin{abstract}
Statistical properties of energy levels and eigenfunctions in a ballistic system with diffusive surface scattering are investigated. The two-level correlation function, the level number variance, the correlation function of wavefunction intensities, and the inverse participation ratio are calculated.

PACS numbers: 05.45.+b, 73.23.Ps, 73.20.Dx
\end{abstract}

The statistical properties of spectra of disordered diffusive systems are now well understood. Using the supersymmetric $\sigma$-model approach it has been possible to demonstrate the relevance of the random matrix theory (RMT) and to calculate deviations from its predictions both for the level [1] 3] and eigenfunction [4 6] statistics. Generalization of these results to the case of a chaotic ballistic system (i.e. quantum billiard) has become a topic of great research interest. For ballistic disordered systems the $\sigma$-model has been proposed [7], with the Liouville operator replacing the diffusion operator in the action. It has also been conjectured that the same $\sigma$-model in the limit of vanishing disorder adequately describes statistical properties of spectra of individual classically chaotic system. This conjecture was further developed in [8,9] where the $\sigma$-model was obtained by means of energy averaging, the Liouville operator replaced by its regularization - the Perron-Frobenius operator and some necessary conditions for validity of this description were put forward.

However, straightforward application of the results of Refs. [2, 4, 6] to the case of an individual chaotic system is complicated by the fact that the eigenvalues of the Perron-Frobenius operator are unknown, while its eigenfunctions are extremely singular. For this reason the $\sigma$ model approach has so far failed to provide explicit results for any particular ballistic system.

To overcome this difficulty, we consider a model of a billiard with surface disorder leading to diffusive scattering of a particle in each collision with the boundary. This models behavior of a quantum particle in a box with a rough boundary which is irregular on the scale of the wave length. Since the particle loses memory of its direction of motion after a single collision, this model describes a limit of an "extremely chaotic" ballistic system, with the typical relaxation time being of order of the flight time. (This should be contrasted with the case of a relatively slight distortion of an integrable billiard [10].) One might naively think that all results for such a model could be obtained by setting $l \approx L$ in a system with bulk disorder. In fact, the level statistics in a system with bulk disorder and arbitrary relation between mean free path $l$ and system size $L$ were studied in 111. We will see, however, that our results are qualitatively different in some respects, which shows that systems with bulk and surface disorder are not equivalent. On the other hand, our findings are in agreement with general expectations for chaotic billiards based on a semiclassical (trace formula) treatment 12.

To simplify the calculations, we consider a circular billiard. A similar problem was studied numerically in Ref. 113 for a square geometry. We consider only the case of unitary symmetry (broken time-reversal invariance); generalization to the orthogonal case is straightforward and will be given elsewhere [14]. The level statistics for the same problem were independently studied in Ref. 15.

Properties of the Liouville operator. Our starting point is the sigma-model derived recently [7] for ballistic disordered systems. The effective action for this model has the form

$$
\begin{aligned}
F[g(\boldsymbol{r}, \boldsymbol{n})] & =\frac{\pi \nu}{4} \int d \boldsymbol{r} \operatorname{Str}\left[i \omega \Lambda\langle g(\boldsymbol{r})\rangle-\frac{1}{2 \tau(\boldsymbol{r})}\langle g(\boldsymbol{r})\rangle^{2}\right. \\
& \left.-2 v_{F}\left\langle\Lambda U^{-1} \boldsymbol{n} \nabla U\right\rangle\right] .
\end{aligned}
$$

Here $g(\boldsymbol{r}, \boldsymbol{n})$ is a $8 \times 8$ supermatrix, which depends on the coordinate $\boldsymbol{r}$ and direction of the momentum $\boldsymbol{n}$. The angular braces denote averaging over $\boldsymbol{n}:\langle\mathcal{O}(\boldsymbol{n})\rangle=$ $\int d \boldsymbol{n} \mathcal{O}(\mathbf{n})$ with the normalization $\int d \boldsymbol{n}=1$. The matrix $g$ is constrained by the condition $g(\boldsymbol{r}, \boldsymbol{n})^{2}=1$, and can be represented as $g=U \Lambda U^{-1}$, with $\Lambda=$ $\operatorname{diag}(1,1,1,1,-1,-1,-1,-1)$; see [1].9] for more detailed definitions. Since we are interested in the clean limit with no disorder in the bulk, the second term in the action (11) containing the elastic mean free time $\tau$ is zero everywhere except at the boundary where it modifies the boundary condition (see below).

Most of the statistical properties of energy levels [16,2,3] and eigenfunctions [4,6] are determined by the structure of the action in the vicinity of the homogeneous configuration of the $g$-field, $g(\boldsymbol{r}, \boldsymbol{n})=\Lambda$. Writing $U=1-W / 2+\ldots$, one finds the action in the leading order in $W$ to be

$$
F_{0}[W]=-\frac{\pi \nu}{4} \int d \boldsymbol{r} d \boldsymbol{n} \operatorname{Str}\left[W_{21}(\hat{K}-i \omega) W_{12}\right],
$$


where the indices 1,2 refer to the "advanced-retarded" decomposition of $W$, and the Liouville operator $\hat{K} \equiv$ $v_{F} \boldsymbol{n} \nabla$. This "linearized" action has the same form as that of a diffusive system, with the diffusion operator being replaced by the Liouville operator. This enables us to use the results derived for the diffusive case by substituting the eigenvalues and eigenfunctions of the operator $\hat{K}$ for those of the diffusion operator.

The operator $\hat{K}$ should be supplemented by a boundary condition, which depends on the form of the surface roughness. As a model approximation we consider purely diffusive scattering [17] for which the distribution function $\varphi(\boldsymbol{r}, \boldsymbol{n})$ of the outgoing particles is constant and is fixed by flux conservation:

$$
\varphi(\boldsymbol{r}, \boldsymbol{n})=\pi \int_{\left(\boldsymbol{N} \boldsymbol{n}^{\prime}\right)>0}\left(\boldsymbol{N} \boldsymbol{n}^{\prime}\right) \varphi\left(\boldsymbol{r}, \boldsymbol{n}^{\prime}\right) d \boldsymbol{n}^{\prime}, \quad(\boldsymbol{N} \boldsymbol{n})<0 .
$$

Here the point $\boldsymbol{r}$ lies at the surface, and $\boldsymbol{N}$ is an outward normal to the surface. This boundary condition should be satisfied by the eigenfunctions of $\hat{K}$.

The eigenvalues $\lambda$ of the operator $\hat{K}$ corresponding to angular momentum $l$ obey the equation

$$
\tilde{J}_{l}(\xi) \equiv-1+\frac{1}{2} \int_{0}^{\pi} d \theta \sin \theta \exp [2 i l \theta+2 \xi \sin \theta]=0,
$$

where $\xi \equiv R \lambda / v_{F}$, and $R$ is the radius of the circle. For each value of $l=0, \pm 1, \pm 2, \ldots$ Eq.(3) has a set of solutions $\xi_{l k}$ with $\xi_{l k}=\xi_{-l, k}$ and $\xi_{l k}=\xi_{l,-k}^{*}$. These $\xi_{l k}$ can be naturally labeled with $k=0, \pm 1, \pm 2, \ldots$ (even $l$ ) or $k= \pm 1 / 2, \pm 3 / 2, \ldots$ (odd $l$ ). For $l=k=0$ we have $\xi_{00}=0$, corresponding to the zero mode $\varphi(\boldsymbol{r}, \boldsymbol{n})=$ const. All other eigenvalues have positive real part $\operatorname{Re} \xi_{l k}>0$ and govern the relaxation of the corresponding classical system to the homogeneous distribution in the phase space.

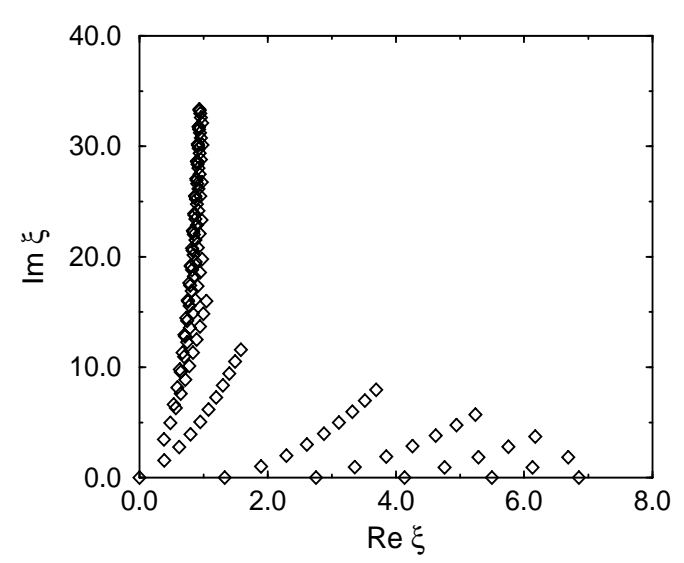

FIG. 1. First $11 \times 11(0 \leq k, l<11)$ eigenvalues of the Liouville operator $\hat{K}$ in units of the inverse time of flight, $v_{F} / R$.

The asymptotic form of the solutions of Eq.(3) for large $|k|$ and/or $|l|$ can be obtained by using the saddle-point method,

$$
\xi_{k l} \approx\left\{\begin{array}{ll}
0.66 l+0.14 \ln l+0.55 \pi i k, & 0 \leq k \ll l \\
(\ln k) / 4+\pi i(k+1 / 8), & 0 \leq l \ll k
\end{array} .\right.
$$

Note that for $k=0$ all eigenvalues are real, while for high values of $k$ they lie close to the imaginary axis and do not depend on $l$ (see Fig. 1).

Level statistics, low frequencies. We define the level correlation function in a standard way,

$$
R_{2}(\omega)=(\Delta V)^{2}\langle\nu(\epsilon+\omega) \nu(\epsilon)\rangle-1,
$$

where $\nu(\epsilon)$ is the density of states, $\Delta=(V \nu)^{-1}$ is the mean level spacing and $V=\pi R^{2}$ is the area. In the range of relatively low frequencies (which for our problem means $\omega \ll v_{F} / R$, see below) the function $R_{2}(\omega)$ quite generally has the form [2]

$$
\begin{aligned}
R_{2}(s) & =\delta(s)-(\pi s)^{-2} \sin ^{2} \pi s \\
& +A\left(R \Delta / \pi v_{F}\right)^{2} \sin ^{2} \pi s
\end{aligned}
$$

where $s=\omega / \Delta$. The first two terms correspond to the zero-mode approximation and are given by RMT, while the last one represents the non-universal correction to the RMT results. It contains information about the operator $\hat{K}$ through the dimensionless constant $A=\sum^{\prime} \xi_{k l}^{-2}$, where the prime indicates that the eigenvalue $\xi_{00}=0$ is excluded. The value of $A$, as well as the high-frequency behavior of the level correlator (see below), can be found from the Altshuler-Shklovskii type spectral function [16]:

$$
S(\omega)=\sum_{l} S_{l}(\omega) ; \quad S_{l}(\omega) \equiv \sum_{k}\left(\lambda_{k l}-i \omega\right)^{-2} .
$$

According to the Cauchy theorem, $S_{l}$ can be represented as an integral in the complex plane,

$$
S_{l}(\omega)=\left(\frac{R}{v_{F}}\right)^{2} \frac{1}{2 \pi i} \oint_{C} \frac{1}{\left(z-i \omega R / v_{F}\right)^{2}} \frac{\tilde{J}_{l}^{\prime}(z)}{\tilde{J}_{l}(z)} d z,
$$

where the contour $C$ encloses all zeroes of the function $\tilde{J}_{l}(z)$. Evaluating the residue at $z=i \omega R / v_{F}$, we find

$$
S_{l}(\omega)=-\left.\left(R / v_{F}\right)^{2} \frac{d^{2}}{d z^{2}}\right|_{z=i \omega R / v_{F}} \ln \tilde{J}_{l}(z) .
$$

Considering the limit $\omega \rightarrow 0$ and subtracting the contribution of $\lambda_{00}=0$, we get

$$
A=-19 / 27-175 \pi^{2} / 1152+64 /\left(9 \pi^{2}\right) \approx-1.48 .
$$

In contrast to the diffusive case, this constant is negative: the level repulsion is enhanced with respect to result for RMT. Eq.(5) is valid as long as the correction is small compared to the RMT result, i.e. provided $\omega$ is below the inverse time of flight, $v_{F} / R$, which plays the role of the Thouless energy for our problem. 
Level statistics, high frequencies. In the range $\omega \gg \Delta$ the level correlation function can be decomposed into the smooth Altshuler-Shklovskii part $R_{2}^{\mathrm{AS}}(\omega)=$ $\left(\Delta^{2} / 2 \pi^{2}\right) \operatorname{Re} S(\omega) \sqrt[16]{ }$ and the part $R_{2}^{\text {osc }}$ which oscillates on the scale of the level spacing. Evaluating the asymptotic behavior of $S_{l}(\omega)$ from Eq.(7), we find in the highfrequency regime when $\omega \gg v_{F} / \vec{R}$ :

$$
R_{2}^{\mathrm{AS}}(\omega)=\left(\frac{\Delta R}{v_{F}}\right)^{2}\left(\frac{v_{F}}{2 \pi \omega R}\right)^{1 / 2} \cos \left(4 \frac{\omega R}{v_{F}}-\frac{\pi}{4}\right) .
$$

The oscillating part of the level correlation function $R_{2}^{\mathrm{OSC}}(s)$ for frequencies $\omega \gg \Delta$ is given by [8]

$$
R_{2}^{\mathrm{OSC}}(s)=\left(1 / 2 \pi^{2}\right) \cos (2 \pi s) D(s),
$$

where $D(s)$ is the spectral determinant,

$$
D(s)=s^{-2} \prod_{k l \neq(00)}\left(1-i s \Delta / \lambda_{k l}\right)^{-1}\left(1+i s \Delta / \lambda_{k l}\right)^{-1} .
$$

Since $\Delta^{-2} \partial^{2} \ln D(s) / \partial s^{2}=-2 \operatorname{Re} S(\omega)$, we can restore $D(s)$ from Eqs.(5), (7)), up to a factor of the form $\exp \left(c_{1}+\right.$ $c_{2} s$ ), with $c_{1}$ and $c_{2}$ being arbitrary constants. These constants are fixed by the requirement that Eq.(10) in the range $\Delta \ll \omega \ll v_{F} / R$ should reproduce the lowfrequency behavior (5). As a result, we obtain

$$
D(s)=\left(\frac{\pi}{2}\right)^{6} \frac{1}{N} \prod_{l} \frac{1}{\tilde{J}_{l}\left(i s N^{-1 / 2}\right) \tilde{J}_{l}\left(-i s N^{-1 / 2}\right)} .
$$

For high frequencies $\omega \gg v_{F} / R$ this yields the following expression for the oscillating part of the level correlation function:

$$
R_{2}^{\mathrm{OSC}}(\omega)=\frac{\pi^{4}}{128}\left(\frac{\Delta R}{v_{F}}\right)^{2} \cos \left(\frac{2 \pi \omega}{\Delta}\right) .
$$

It is remarkable that the amplitude of the oscillating part does not depend on frequency. This is in contrast to the diffusive case, where in the Altshuler-Shklovskii regime ( $\omega$ above the Thouless energy) the oscillating part $R_{2}^{\mathrm{OSC}}(\omega)$ is exponentially small [3].

The level number variance. The smooth part of the level correlation function can be best illustrated by plotting the variance of the number of levels in an energy interval of width $E=s \Delta$,

$$
\Sigma_{2}(s)=\int_{-s}^{s}(s-|\tilde{s}|) R_{2}(\tilde{s}) d \tilde{s},
$$

A direct calculation gives the following asymptotic behavior:

$$
\pi^{2} \Sigma_{2}(s)=1+\gamma+\ln (2 \pi s)+A s^{2} /(2 N)
$$

when $s \ll N^{1 / 2}$ and

$$
\begin{aligned}
\pi^{2} \Sigma_{2}(s)= & +\gamma+\ln \frac{16 N^{1 / 2}}{\pi^{2}} \\
& -\frac{\pi^{2}}{16}\left(\frac{2 N^{1 / 2}}{\pi s}\right)^{1 / 2} \cos \left(\frac{4 s}{N^{1 / 2}}-\frac{\pi}{4}\right)
\end{aligned}
$$

when $s \gg N^{1 / 2}$. Here $N=\left(v_{F} / R \Delta\right)^{2}=\left(p_{F} R / 2\right)^{2}$ is the number of electrons below the Fermi level, $\gamma \approx 0.577$ is Euler's constant, and $A$ is defined by Eq.(8). The first three terms at the rhs of Eq. (14) represent the RMT contribution (curve 1 in Fig. 2). The two asymptotics (14) and (15) are shown in Fig. 1 as curves 2 and 3 respectively.

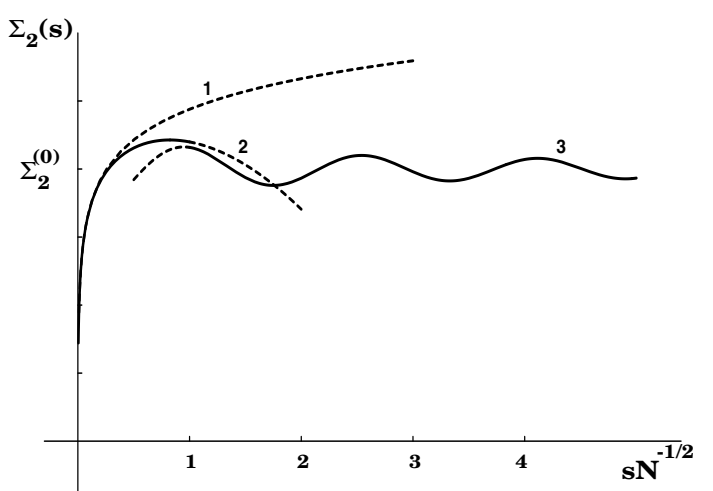

FIG. 2. Level number variance $\Sigma_{2}(E)$ as a function of energy; $s=E / \Delta$. Curve 1 shows the RMT result, while curves 2 and 3 correspond to asymptotic regimes of low (14) and high (15) frequencies. The saturation value $\Sigma_{2}^{(0)}$ is given in the text.

As seen from Fig.2, the two curves (14) and (15) perfectly match in the intermediate regime, $s \sim N^{1 / 2}$, and taken together they provide a complete description of $\Sigma_{2}(s)$. According to Eq.(15), the level number variance saturates at the value $\Sigma_{2}^{(0)}=\pi^{-2}\left(1+\gamma+\ln \left(16 N^{1 / 2} / \pi^{2}\right)\right)$, in contrast to the behavior found for diffusive systems 16 or ballistic systems with weak bulk disorder 111. The saturation occurs at energies $s \sim N^{1 / 2}$, or in conventional units $E \sim v_{F} / R$. This behavior of $\Sigma_{2}(s)$ is expected for a generic chaotic billiard [12]. It is also in good agreement with the results for $\Sigma_{2}(s)$ found numerically for a tight-binding model with moderately strong disorder on boundary sites 13.

Eigenfunction statistics. Now we study correlations of the amplitudes of an eigenfunction in two different points. Correlation of different eigenfunctions will be considered elsewhere [14]. Following Ref. [6], we define

$$
\alpha\left(\boldsymbol{r}_{\mathbf{1}}, \boldsymbol{r}_{\mathbf{2}}, E\right)=\Delta V^{2}\left\langle\sum_{\mu}\left|\psi_{\mu}\left(\boldsymbol{r}_{\mathbf{1}}\right) \psi_{\mu}\left(\boldsymbol{r}_{\mathbf{2}}\right)\right|^{2} \delta\left(E-\epsilon_{\mu}\right)\right\rangle,
$$

where $\psi_{\mu}$ are the eigenfunctions corresponding to the exact single-particle states $\mu$. A calculation analogous to that of Ref. [6] yields

$$
\alpha\left(\boldsymbol{r}_{\mathbf{1}}, \boldsymbol{r}_{\mathbf{2}}, E\right)=1+\Pi\left(\boldsymbol{r}_{\mathbf{1}}, \boldsymbol{r}_{\mathbf{2}}\right),
$$


where $\Pi$ is the Green's function of the operator $\hat{K}$ integrated over directions of momentum, $\Pi\left(\boldsymbol{r}_{\mathbf{1}}, \boldsymbol{r}_{\mathbf{2}}\right)=$ $\int d \boldsymbol{n}_{\mathbf{1}} d \boldsymbol{n}_{\mathbf{2}} g\left(\boldsymbol{r}_{\mathbf{1}}, \boldsymbol{n}_{\mathbf{1}} ; \boldsymbol{r}_{\mathbf{2}}, \boldsymbol{n}_{\mathbf{2}}\right)$. Here $g$ is the full Green's function of the operator $\hat{K}$, i.e. a solution to the equation

$$
\begin{aligned}
& \hat{K} g\left(\boldsymbol{r}_{\mathbf{1}}, \boldsymbol{n}_{\mathbf{1}} ; \boldsymbol{r}_{\mathbf{2}}, \boldsymbol{n}_{\mathbf{2}}\right)= \\
& (\pi \nu)^{-1}\left[\delta\left(\boldsymbol{r}_{\mathbf{1}}-\boldsymbol{r}_{\mathbf{2}}\right) \delta\left(\boldsymbol{n}_{\mathbf{1}}-\boldsymbol{n}_{\mathbf{2}}\right)-V^{-1}\right] .
\end{aligned}
$$

Direct calculation gives:

$$
\begin{aligned}
& \Pi\left(\boldsymbol{r}_{\mathbf{1}}, \boldsymbol{r}_{\mathbf{2}}\right)=\Pi_{1}\left(\boldsymbol{r}_{\mathbf{1}}, \boldsymbol{r}_{\mathbf{2}}\right)+\Pi_{2}\left(\boldsymbol{r}_{\mathbf{1}}, \boldsymbol{r}_{\mathbf{2}}\right), \\
& \Pi_{1}\left(\boldsymbol{r}_{\mathbf{1}}, \boldsymbol{r}_{\mathbf{2}}\right)=\tilde{k}_{d}\left(\boldsymbol{r}_{\mathbf{1}}-\boldsymbol{r}_{\mathbf{2}}\right)-V^{-1} \int d \boldsymbol{r}_{\mathbf{1}}^{\prime} \tilde{k}_{d}\left(\boldsymbol{r}_{\mathbf{1}}^{\prime}-\boldsymbol{r}_{\mathbf{2}}\right) \\
& -V^{-1} \int d \boldsymbol{r}_{\mathbf{2}}^{\prime} \tilde{k}_{d}\left(\boldsymbol{r}_{\mathbf{1}}-\boldsymbol{r}_{\mathbf{2}}^{\prime}\right)+V^{-2} \int d \boldsymbol{r}_{\mathbf{1}}^{\prime} d \boldsymbol{r}_{\mathbf{2}}^{\prime} \tilde{k}_{d}\left(\boldsymbol{r}_{\mathbf{1}}^{\prime}-\boldsymbol{r}_{\mathbf{2}}^{\prime}\right) ; \\
& \Pi_{2}\left(\boldsymbol{r}_{\mathbf{1}}, \boldsymbol{r}_{\mathbf{2}}\right)=\frac{1}{4 \pi p_{F} R} \sum_{k=1}^{\infty} \frac{4 k^{2}-1}{4 k^{2}}\left(\frac{r_{1} r_{2}}{R^{2}}\right)^{k} \cos k\left(\theta_{1}-\theta_{2}\right)
\end{aligned}
$$

where $\tilde{k}_{d}(\boldsymbol{r})=1 /\left(\pi p_{F}|\boldsymbol{r}|\right)$, and $(r, \theta)$ are the polar coordinates. This formula has a clear interpretation. The function $\Pi$ can be represented as a sum over all possible paths leading from $\boldsymbol{r}_{\mathbf{1}}$ to $\boldsymbol{r}_{\mathbf{2}}$, with possible surface scattering in between. In particular, the function $\Pi_{1}$ corresponds to trajectories coming directly from $\boldsymbol{r}_{\mathbf{1}}$ to $r_{2}$ with no reflection from the surface. Therefore, the term $\Pi_{1}$ is universal and is not sensitive to the geometry of the system. It can be obtained from the RMT-like prediction that amplitudes of different wavefunctions are independent Gaussian variables [18]. More precisely, we find that the function $k_{d}\left(\mathbf{r}_{\mathbf{1}}-\mathbf{r}_{\mathbf{2}}\right)=J_{0}^{2}\left(p_{F}\left|\mathbf{r}_{\mathbf{1}}-\mathbf{r}_{\mathbf{2}}\right|\right)$ of Refs. [6.18] is replaced in Eq. (18) by its smoothed version, $k_{d}\left(\mathbf{r}_{\mathbf{1}}-\mathbf{r}_{\mathbf{2}}\right)=1 /\left(\pi p_{F}\left|\mathbf{r}_{\mathbf{1}}-\mathbf{r}_{\mathbf{2}}\right|\right)$. This is because our semiclassical approach is valid on scales much larger than the wave length. To cure this flaw, one has to replace $\tilde{k}_{d}(\mathbf{r})$ by $k_{d}(\mathbf{r})$ in the expression Eq. (18) for $\Pi_{1}$. The second term, $\Pi_{2}$, is due to the surface scattering. It can be shown [14] that in the numerator $4 k^{2}-1$ the first term comes from trajectories with only one surface reflection, while the second sums up contributions from multiple reflections. A formula analogous to (16) was proposed very recently for a generic chaotic system 19$]$.

Finally, we calculate the inverse participation ratio, $\left\langle P_{2}\right\rangle \equiv V^{-2} \int d \boldsymbol{r} \alpha(\boldsymbol{r}, \boldsymbol{r})$, which characterizes the degree of spatial uniformity of eigenfunctions. The RMT prediction for this quantity, $P_{2}^{(0)}=2 / V$ is recovered from Eqs.(16), (18) if we take into account the first term in the expression for $\Pi_{1}$, since $k_{d}(0)=1$. The leading correction comes from the single-reflection contribution to the term $\Pi_{2}$, and is equal to

$$
\delta P_{2}=V^{-1}\left(4 \pi p_{F} R\right)^{-1} \ln \left(p_{F} R\right) \sim P_{2}^{(0)} N^{-1 / 2} \ln N .
$$

In conclusion, we have used the ballistic $\sigma$-model approach to study statistical properties of levels and eigenfunctions in a billiard with diffusive surface scattering, which exemplifies a ballistic system in the regime of strong chaos. We have found that the level repulsion and the spectral rigidity are enhanced compared to RMT. In particular, the level number variance saturates at the scale of the inverse time of flight, in agreement with Berry's prediction for a generic chaotic system [12]. As another manifestation of the strong spectral rigidity, the oscillating part of the level correlation function does not vanish at large level separation. We have also considered correlations of eigenfunction amplitudes in different spatial points and calculated the deviation of the inverse participation ratio from the RMT value.

This work was supported by the Swiss National Science Foundation and SFB 195 der Deutschen Forschungsgemeinschaft. We are grateful to the University of Geneva (A. D. M.) and Isaac Newton Institute for Mathematical Sciences of the Cambridge University, where part of this work was done, for hospitality and support.

* Also at St.Petersburg Nuclear Physics Institute, 188350 St.Petersburg, Russia.

[1] K. B. Efetov, Adv. Phys. 32, 53 (1983).

[2] V. E. Kravtsov and A. D. Mirlin, Pis'ma Zh. Éksp. Teor. Fiz. 60, 645 (1994) [JETP Lett. 60656 (1994)].

[3] A. V. Andreev and B. L. Altshuler, Phys. Rev. Lett. 75, 902 (1995).

[4] Y. V. Fyodorov and A. D. Mirlin, Phys. Rev. B 51, 13403 (1995).

[5] V. I. Fal'ko and K. B. Efetov, Phys. Rev. B 52, 17413 (1995).

[6] Ya. M. Blanter and A. D. Mirlin, Phys. Rev. E 55, 6514 (1997).

[7] B. A. Muzykantskii and D. E. Khmelnitskii, Pis'ma Zh. Éksp. Teor. Fiz. 6268 (1995) [JETP Lett. 62, 76 (1995)].

[8] O. Agam, B. L. Altshuler, and A. V. Andreev, Phys. Rev. Lett. 75, 4389 (1995).

[9] A. V. Andreev et al, Phys. Rev. Lett. 76, 3947 (1996); Nucl.Phys. B 482, 536 (1996).

[10] F. Borgonovi, G. Casati, and B. Li, Phys. Rev. Lett. 77, 4744 (1996); K. M. Frahm and D. L. Shepelyansky, ibid 78, 1440 (1997); ibid 79, 1833 (1997).

[11] A. Altland and Y. Gefen, Phys. Rev. Lett. 71, 3339 (1992); O. Agam and S. Fishman, J. Phys. A 29, 2013 (1996).

[12] M. V. Berry, Proc. R. Soc. London A 400, 229 (1985).

[13] E. Louis et al, Phys. Rev. B 56, 2120 (1997).

[14] Ya. M. Blanter, A. D. Mirlin, and B. A. Muzykantskii (unpublished).

[15] V. Tripathi and D. E. Khmelnitskii (unpublished).

[16] B. L. Altshuler and B. I. Shklovskii, Zh. Éksp. Theor. Fiz. 91, 220 (1986) [Sov. Phys. JETP 64, 127 (1986)].

[17] K. Fuchs, Proc. Cambridge Phil. Soc. 34, 100 (1938); Yu. N. Ovchinnikov, Zh. Éksp. Theor. Fiz. 56, 1590 (1969) [Sov. Phys. JETP 29, 853 (1969)]

[18] M. V. Berry, J. Phys. A 10, 2083 (1977); V. N. Prigodin, Phys. Rev. Lett. 74, 1566 (1995); M. Srednicki, Phys. Rev. E 54, 954 (1996).

[19] S. Hortikar and M. Srednicki, chao-dyn/9710025 (unpublished). 